\title{
Effectiveness of complete primary vaccination against COVID-19 at primary care and community level during predominant Delta circulation in Europe: multicentre study analysis by age-group, vaccine brand and time since vaccination, I-MOVE- COVID-19 and ECDC networks, July-August 2021
}

Authors: Esther Kissling ${ }^{1}$, Mariëtte Hooiveld ${ }^{2}$, Iván Martínez-Baz ${ }^{3,4}$, Clara Mazagatos ${ }^{5,4}$, Naoma William ${ }^{6}$, Ana-Maria Vilcu ${ }^{7}$, Marjolein N. Kooijman ${ }^{8}$, Maja Ilić ${ }^{9}$, Lisa Domegan ${ }^{10}$, Ausenda Machado $^{11}$, Simon de Lusignan ${ }^{12,13}$, Mihaela Lazar ${ }^{14}$, Adam Meijer ${ }^{8}$, Mia Brytting ${ }^{15}$, Itziar Casado ${ }^{3,4}$, Amparo Larrauri ${ }^{5,4}$, Josephine-L K. Murray ${ }^{6}$, Sylvie Behillil ${ }^{16,17}$, Brechje de Gier ${ }^{8}$, Ivan Mlinarić ${ }^{9}$, Joan $\mathrm{O}^{\prime}$ Donnell ${ }^{10}$, Ana Paula Rodrigues ${ }^{11}$, Ruby Tsang ${ }^{12,13}$, Olivia Timnea ${ }^{14}$, Marit de Lange ${ }^{8}$, Maximilian Riess $^{15}$, Jesús Castilla ${ }^{3,4}$, Francisco Pozo ${ }^{18}$, Mark Hamilton ${ }^{6}$, Alessandra Falchi ${ }^{19}$, Mirjam J. Knol ${ }^{8}$, Sanja Kurečić Filipović ${ }^{9}$, Linda Dunford ${ }^{20}$, Raquel Guiomar ${ }^{11}$, Jade Cogdale ${ }^{21}$, Carmen Cherciu ${ }^{14}$, Tessa Jansen ${ }^{2}$, Theresa Enkirch ${ }^{15}$, Luca Basile ${ }^{4,22}$, Jeff Connell ${ }^{20}$, Verónica Gomez ${ }^{11}$, Virginia Sandonis Martín ${ }^{18}$, Sabrina Bacci ${ }^{23}$, Angela MC Rose ${ }^{1}$, Lucia Pastore Celentano ${ }^{23}$, Marta Valenciano ${ }^{1}$, I-MOVE-COVID-19 and ECDC primary care study teams.

\section{Affiliations}

1. Epiconcept, Paris, France

2. Nivel, the Netherlands

3. Instituto de Salud Pública de Navarra - IdiSNA, Pamplona, Spain

4. Consortium for Biomedical Research in Epidemiology and Public Health (CIBERESP), Madrid, Spain

5. National Centre for Epidemiology, Institute of Health Carlos III, Madrid, Spain

6. Public Health Scotland, Glasgow, Scotland

7. INSERM, Sorbonne Université, Institut Pierre Louis d'épidémiologie et de Santé Publique (IPLESP UMRS 1136), Paris, France

8. National Institute for Public Health and the Environment (RIVM), Bilthoven, the Netherlands

9. Croatian Institute of Public Health, Zagreb, Croatia

10. Health Service Executive-Health Protection Surveillance Centre, Dublin, Ireland

11. Instituto Nacional de Saúde Dr. Ricardo Jorge, Lisbon, Portugal

12. Nuffield Department of Primary Care Health Sciences, University of Oxford, Oxford, UK

13. Royal College of General Practitioners Research and Surveillance Centre, London, UK

14. "Cantacuzino" National Military Medical Institute for Research and Development, Bucharest, Romania

15. The Public Health Agency of Sweden, Stockholm, Sweden

16. Unité de Génétique Moléculaire des Virus à ARN, UMR 3569 CNRS, Université Paris Diderot SPC, Institut Pasteur, Paris, France

17. CNR des virus des infections respiratoires, Institut Pasteur, Paris, France

18. National Centre for Microbiology, Institute of Health Carlos III, Madrid, Spain

19. Laboratoire de Virologie, Université de Corse-Inserm, Corte, France

20. National Virus Reference Laboratory, University College Dublin, Dublin, Ireland

21. Public Health England, London, England

22. Subdirección General de Vigilancia y Respuesta a Emergencias de Salud Pública, Agencia de Salud Pública, Catalunya, Spain

23. European Centre for Disease Prevention and Control, Stockholm, Sweden 


\section{Abstract}

\section{Introduction}

In July and August 2021, the SARS-CoV-2 Delta variant dominated in Europe. We measured COVID19 vaccine effectiveness (VE) against symptomatic infection, using a multicentre test-negative study at primary care/community level in Europe.

\section{Methods}

Patients presenting with COVID-19/ARI symptoms at primary care/community level in 10 countries were tested for SARS-CoV-2. We measured complete primary course overall VE among those aged $30-44,45-59,60-74$ and $\geq 75$ years, and among those $30-59$ and $\geq 60$ years by vaccine brand and by time since vaccination.

\section{Results}

Overall VE was 74\% (95\%Cl: 69-79), 76\% (95\%Cl: 71-80), 63\% (95\%Cl: 48-75), 63\% (95\%Cl: 16-83) among those aged $30-44,45-59,60-74$ and $\geq 75$ years, respectively. VE among those aged 30-59 years was 78\% (95\%Cl: 75-81), 66\% (95\%Cl: 58-73), 91\% (95\% Cl: 87-94) and 52\% (95\%Cl: 40-61), for Comirnaty, Vaxzevria, Spikevax and COVID-19 Vaccine Janssen, respectively. VE among those aged $\geq 60$ years was $67 \%$ (95\% Cl: $52-77), 65 \%$ (95\%Cl: $48-76), 83 \%$ (95\%Cl: $64-92)$ for Comirnaty, Vaxzevria and Spikevax, respectively.

Comirnaty VE among those aged $30-59$ years was $87 \%(95 \% \mathrm{Cl}: 83-89)$ and $65 \%(95 \% \mathrm{Cl}: 56-71 \%)$ at 14-29 days and $\geq 90$ days between vaccination and onset of symptoms, respectively.

\section{Conclusions}

VE against the symptomatic SARS-CoV-2 Delta variant infection varied among brands, ranging from 52-91\%. While some waning of the vaccine effect may be present (sample size limited this analysis to only Comirnaty), protection was $65 \% \geq 90$ days between vaccination and onset. 


\section{Introduction}

COVID-19 has caused considerable morbidity and mortality with over 76.7 million cases and 1.4 million deaths reported in Europe (1). The highly transmissible Delta variant (B.1.617.2 and the AY sublineages in the Pango nomenclature system) dominated over the Alpha variant in Europe from July 2021 onwards and accounted for over 99\% of sequenced samples in weeks 35-36 2021 (2-4). From the end of October 2021, four COVID-19 vaccines were authorised in the European Union by the European Medicines Agency: two mRNA vaccines (Comirnaty and Spikevax), and two adenoviral vector vaccines (Vaxzevria and COVID-19 Vaccine Janssen)(5). Prior to the Delta circulation, randomised controlled trials indicated a high efficacy for these vaccines (6-9). Observational post-authorisation studies in Europe are therefore important to measure the effectiveness of the different COVID-19 vaccines against the currently circulating Delta variant. Recent discussions around recommendations for booster doses, for various populations, highlight the need to measure vaccine effectiveness (VE) by time since vaccination. Some studies have reported a decrease in VE against infection with increasing time since vaccination, but not all (1016). It may be difficult to disentangle potential changes in VE by time since vaccination from changes in VE due to circulation of different variants over time.

The I-MOVE-COVID-19 and ECDC networks in Europe carry out COVID-19 VE studies, including a VE study at primary care/community level (17). All studies are based on a common generic protocol (18).

We conducted a study in symptomatic patients swabbed at primary and care/community level in July and August 2021, assuming SARS-CoV-2 cases were of the Delta variant due to its dominance in Europe during this period. We estimated VE against SARS-CoV-2 symptomatic infection, by vaccine brand, age group, and by time since vaccination to understand if there may be waning of protection conferred by vaccines over time.

\section{Methods}

\section{Study design}

Ten of 14 I-MOVE-COVID-19 primary care/community study sites participated in this analysis: Croatia (HR), France (FR), Ireland (IE), the Netherlands (community testing: NL-CO), Portugal (PT), 
Romania (RO), Spain (3 regions: ES; Navarra region: NA), and the UK (England: EN; Scotland: SC) (Figure 1).

Figure 1: Countries and study sites taking part in the I-MOVE-COVID-19 and ECDC VE studies at primary care/community level

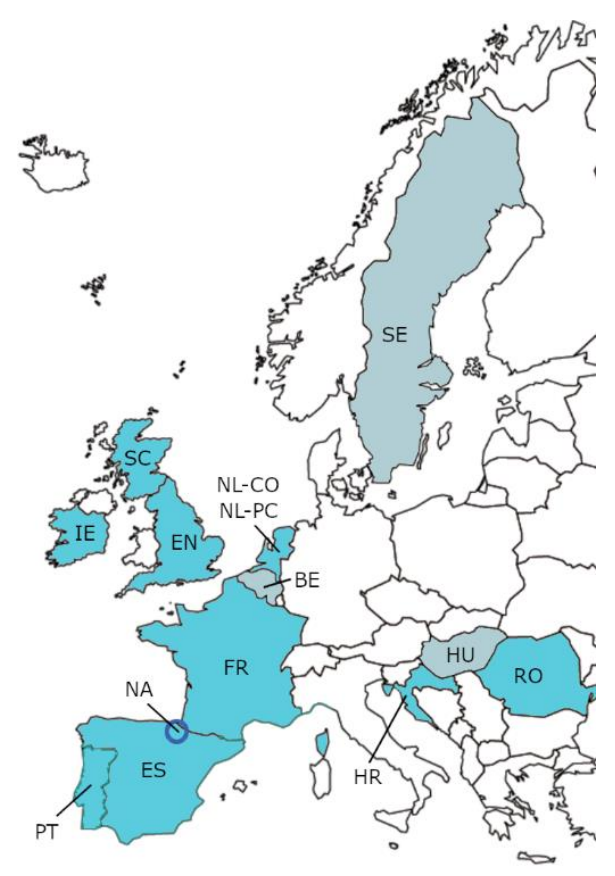

Included in the current analysis

Not included in the current analysis

BE: Belgium; EN: England; ES: Spain; FR: France; HR: Croatia; HU: Hungary; IE: Ireland; NL-CO: the Netherlands community-based study; NL-PC: the Netherlands primary care; PT: Portugal; RO: Romania; SE: Sweden.

We used the test-negative design to estimate VE against symptomatic infection (19). Cases were patients testing positive and controls patients testing negative for SARS-CoV-2. Study sites adapted a generic study protocol to their country-specific setting (18). In eight of the 10 participating study sites a sentinel physician recruited patients. SC uses a combination of physician-based and community-based swabbing, and NL-CO uses a purely community-based swabbing approach. Sites included all or a systematic sample of acute respiratory infection (ARI) patients or patients presenting with COVID-19 symptoms (at least one of the following symptoms: fever, cough, shortness of breath, sudden onset of anosmia/ageusia) who contacted the sentinel physician/swabbing centre (Supplementary table 1). NA included all patients presenting to the Navarra Health Service primary care physicians. SC included a systematic sample of patients 
presenting to community-attended COVID-19 centres, where both self- and clinical staff-based swabbing were performed. In NL-CO, participating patients were self-selected.

Demographic, clinical (age, sex and chronic conditions) and COVID-19 vaccination information (number of doses, brand and date for each dose) was collected, via questionnaire, electronic medical records and vaccine registry linkage (Supplementary table S1).

Where technically feasible, the whole or partial genome of viruses confirmed by PCR was sequenced. Results from four study sites (HR, IE, NA, RO) were available at time of writing. Phylogenetic analysis was performed to identify the Phylogenetic Assignment of Named Global Outbreak (Pango) lineage based on the classification v.3.1.16 2021.

\section{Study period}

The study period included swab dates in July and August 2021, as this is when the Delta variant was dominant in participating study sites $(20,21)$.

\section{Study inclusion criteria}

We included patients aged 30 years and older who belonged to their country's age-specific target group for vaccination at time of swab. Where known, we excluded patients with contraindications for vaccination, living in a residential care facility, controls previously testing positive for SARSCoV-2 and controls testing positive for seasonal coronaviruses. We further excluded those who were swabbed more than 10 days (PCR tests) or 5 days (rapid antigen tests) after symptom onset. We excluded those with a delay between doses not recommended by the vaccine manufacturer (<21 days for Comirnaty, <28 days for Spikevax/Vaxzevria, <21 days if vaccine brand was unknown) and those with onset within 1-13 days of first (if COVID-19 vaccine Janssen) or second dose (for two-dose vaccines) of COVID-19 vaccine.

\section{Definitions of vaccination}

We defined persons as completely vaccinated 14 days after receiving either the second of two recommended doses of a two-dose vaccine or a single dose of COVID-19 Vaccine Janssen. Persons were considered unvaccinated if they did not receive any COVID-19 vaccine or were vaccinated on or after the day of onset of symptoms. Persons partially vaccinated were excluded from the analysis. 
For the brand-specific analysis, we restricted to the period in which the brand was available in the country by age group. For two-dose vaccines, we only included persons vaccinated with the same brand for both doses

\section{Statistical analysis}

We compared the odds of vaccination between cases and controls. We used logistic regression and calculated VE as 1 minus the odds ratio expressed as a percentage. We included study site as a fixed effect and date of swab (modelled as a restricted cubic spline or as a categorical variable of swab week) in both crude and adjusted VE analyses, as time is an integral part of the test-negative design and study site of a multicentre study.

We further adjusted for age group, sex and presence of at least one of the six commonly collected COVID-19 relevant chronic conditions (diabetes, chronic lung disease, immunodeficiencies, heart disease, renal disease and liver disease). In SC, the question "Did you receive a letter asking you to shield?" was used as a proxy for "presence of chronic condition", assuming those not answering the question were not shielding.

In one study site where symptom onset date was not available (NA) and in two study sites with $\geq 20 \%$ of missing information (IE and PT), we imputed it as 3 days before the swab date, three days being the median between onset and swab date where information was available.

For the age-specific analyses for all vaccines, we stratified the data into the following age groups: $30-44,45-59,60-74$ and $\geq 75$ years.

For the analyses among those with a chronic condition, vaccine brand and by time since vaccination, we stratified the data into the age groups $30-59$ and $\geq 60$ years.

We modelled VE by time since vaccination using an interaction between vaccination and time since vaccination as a restricted cubic spline with 4 knots at 0 and 20 days and the $45^{\text {th }}$ and $90^{\text {th }}$ centile, based on a priori knots and adaptations of Harrell's percentiles (22). We also measured VE by days between vaccination and onset of symptoms with stratified by monthly cut-offs: $<30,30-$ $59,60-89$ days and $\geq 90$ days, and superimposed these values on the graph of the model to provide a validation of the modelling. We did not compute VE if one of these strata had less than 100 patients. 
We conducted sensitivity analyses excluding the study site without systematic selection of patients for swabbing (NL-CO), excluding the largest study site (NA), varying the imputed onset dates between 2 and 5 days, including only those swabbed within 7 days of symptom onset (PCR tests), and varying the number and position of knots in the time since vaccination analysis.

\section{Ethics}

The planning, conduct and reporting of the studies was in line with the Declaration of Helsinki. Official ethical approval was not required if studies were classified as being part of routine care or within surveillance regulations: Spain (three regions) regulated by royal decree 2210/1995 of December 28 provided by the Ministry of Health and Consumer Affairs, England and Scotland (Regulation 3 of the Health Service (Control of Patient Information) Regulations 2002 to collect confidential patient information (www.legislation.gov.uk/uksi/2002/1438/regulation/3/made.opensinnewtab) under Sections 3(i) (a) to (c), 3(i)(d) (i) and (ii), and 3), and Ireland (virological and clinical surveillance of COVID-19 is classified as routine operational public health activities conducted in the public interest can therefore be conducted without informed consent under one of the appropriate Article 6 grounds (like public interest) and Article 9(2)(i) of the General Data Protection Regulations (GDPR) (or HSE can avail of sections 38 and 39)). Other study sites obtained local ethical approval from a national review board: France: approved by the French ethics research committee ('Comité de Protection des Personnes' - CPP), no registration number given; Navarra, Spain, approved by Comité de ética de la ivestigación con medicamentos(PI2020/45); Portugal: approved 16 March 2021 by the Ethics Committee of Instituto Nacional de Saúde Doutor Ricardo Jorge, no registration number given; Croatia: approved 17 June 2021 by the Ethics Committee of the Croatian Institute of Public Health, no registration number; the Netherlands (NL-CO): a waiver for ethical review was issued by the National Institute for Public Health and the Environment of the Netherlands, online informed consent was provided by all participants; Romania: the study was approved on 12 of April 2021 by the Cantacuzino Institute ethics committee (CE 47/2021). 


\section{Results}

After applying the exclusion criteria, we included 14,282 patients, of which 2,725 were cases and 11,557 were controls (Supplementary Figure S1).

Among the cases and controls, 11,312 were completely vaccinated. Vaccination rollout continued as cases and controls were selected (Figure 2).

Figure 2: Number of patients by week of swab and case status, as well as number of patients by week of their complete vaccination, I-MOVE-COVID-19 and ECDC primary care and community multicentre networks, July-August 2021

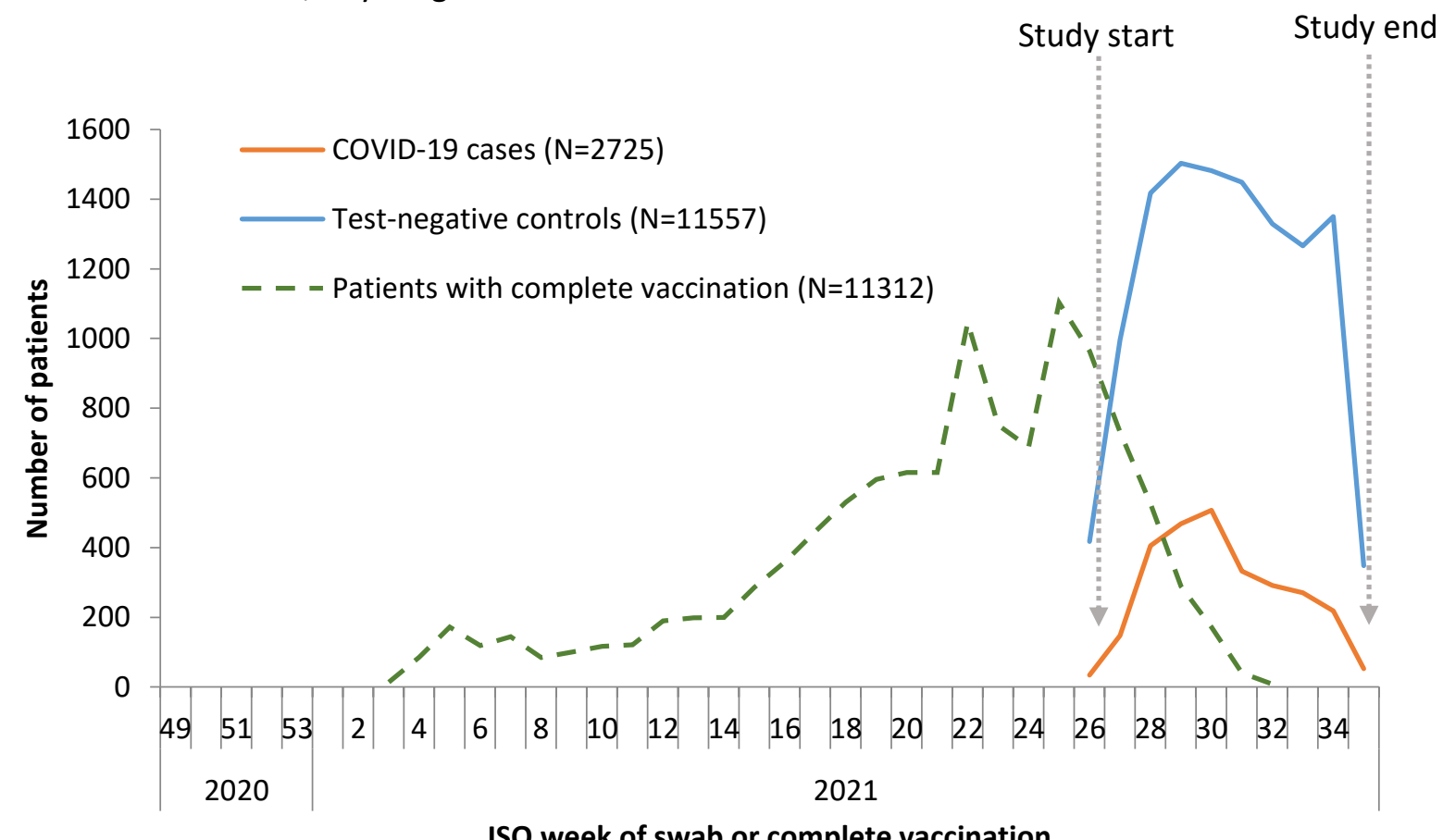

Cases and controls received their second dose (for 2-dose schedules), or first dose (for COVID-19 Vaccine Janssen) of COVID-19 vaccination up to 213 days before symptom onset (Figure 3). 
Figure 3: Completely vaccinated patients by case status and days between complete vaccination and symptom onset, I-MOVE-COVID-19 and ECDC primary care and community multicentre networks, July-August 2021

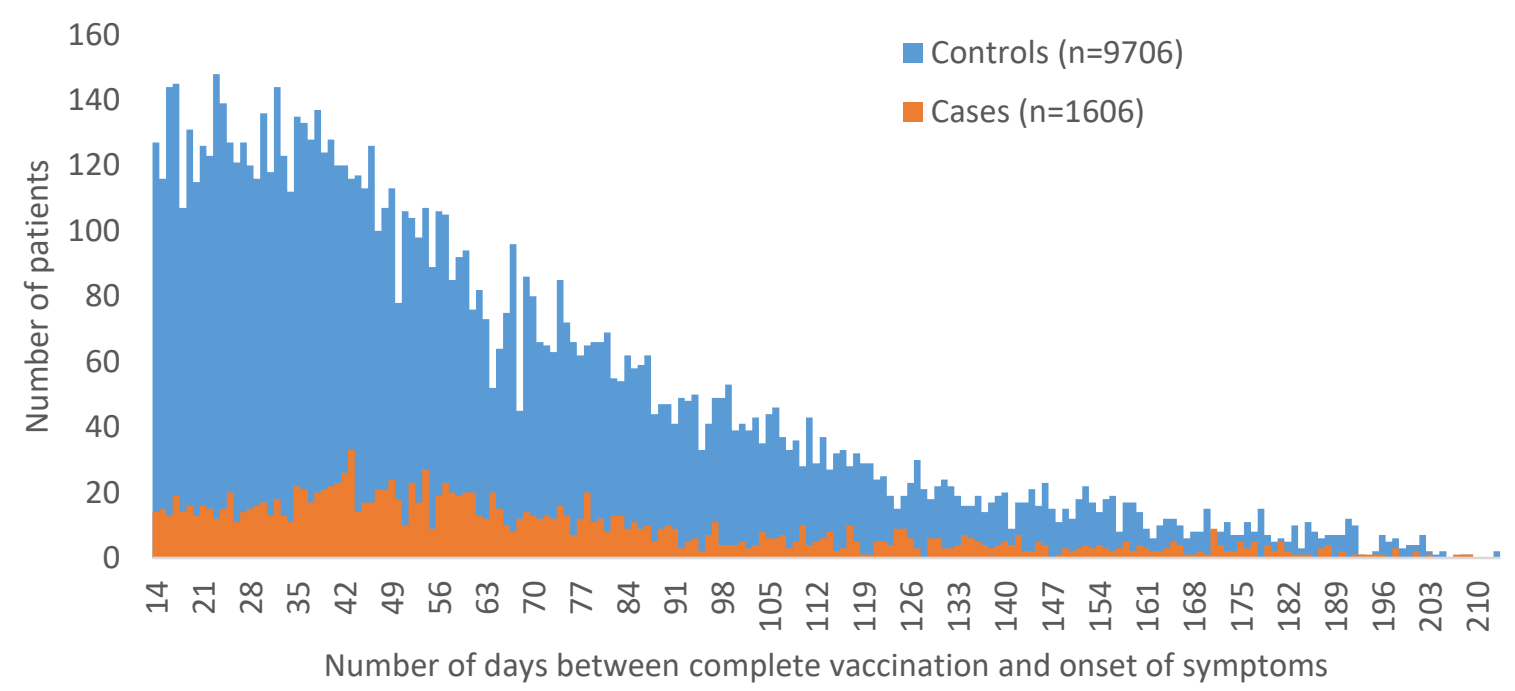

Among controls, $35 \%$ were aged 60 and older compared to $24 \%$ of cases, $59 \%$ were female (vs $54 \%$ of cases), and $31 \%$ (vs $23 \%$ of cases) presented with at least one chronic condition (diabetes, heart disease, chronic lung disease, immunodeficiency, renal disease, or liver disease) (Supplementary Table S2).

A total of $84 \%$ of controls had received complete COVID-19 vaccination at time of swab, compared to $59 \%$ of cases. Comirnaty was the most commonly used vaccine, with $66 \%$ of vaccinated controls and $60 \%$ of vaccinated cases receiving two doses.

Of the 2190 cases presenting in the four study sites with sequencing results available, 130 (6\%) were sequenced and 118 (91\%) of these belonged to the SARS-CoV-2 Delta variant and its sublineages.

The overall complete dose VE against symptomatic SARS-CoV-2 infection was 74\% (95\% Cl: 70-77) among persons aged 30 years and older, adjusting for swab date, study site, age group, sex and chronic condition. The adjusted VE was 74\% (95\% Cl: 69-79), 76\% (95\% Cl: 71-80), 64\% (95\% Cl: 48-75) and 63\% (95\% $\mathrm{Cl} 16-83)$ for those aged 30-44, 45-59, 60-74 and 75 years and older, respectively (Table 2). The VE for two doses of Comirnaty was 78\% (95\% Cl: 75-81) and 67\% (95\% Cl: 52-77) among 30-59-year-olds and those aged 60 and older, respectively. The VE for two doses of Vaxzevria was 66\% (95\% Cl: 58-73) and 65\% (95\% Cl: 48-76) among 30-59-year-olds and those 
aged 60 years and older, respectively. The VE for two doses of Spikevax was 91\% (95\% Cl: 87-94) and $83 \%$ (85\% Cl: 64-92) among 30-59-year-olds and those aged 60 years and older, respectively. The VE of COVID-19 Vaccine Janssen was 52\% (95\% Cl: 40-61) among 30-59-year-olds. VE was not computed for COVID-19 Vaccine Janssen among those aged 60 years and older, as all countries but one dropped out of the analysis.

The overall VE for those aged $30-59$ years with presence of chronic condition was $63 \%(95 \% \mathrm{Cl}$ : 50-73) and without 77\% (95\% Cl: 73-80) (p-value for interaction term 0.007). The VE for those aged 60 years and older with presence of chronic condition was 58\% (95\% Cl: 27-76) and without $66 \%$ (95\% Cl: 49-78). Among those aged $30-59$ years the VE was $69 \%$ (95\% $\mathrm{Cl}: 56-78)$ and $80 \%$ (95\% Cl: 77-83), 50\% (95\% Cl: 11-72) and 68\% (95\% Cl: 60-75), 94\% (95\% Cl: 83-98) and 90\% (95\% Cl: 86-94), and 24\% (95\% Cl: -25-53) and 57\% (95\% Cl: 45-66), for those with and without presence of chronic condition for Comirnaty, Vaxzevria, Spikevax and COVID-19 Vaccine Janssen, respectively (Table 1). Sample size was too small to measure brand-specific VE stratified by presence of chronic condition among those aged 60 years and older.

Table 2: Effectiveness of complete COVID-19 vaccination among participants in the primary care and community I-MOVE-COVID-19 and ECDC VE study, by age group and vaccine product, Europe, July-August 2021

\begin{tabular}{|c|c|c|c|c|}
\hline Vaccine brand & Analysis type & $\begin{array}{l}\text { Cases; vaccinated/ } \\
\text { controls; vaccinated }\end{array}$ & Crude VE $(95 \% \mathrm{Cl})^{\mathrm{a}}$ & $\begin{array}{l}\text { Adjusted } \\
\text { VE }(95 \% \\
\mathrm{Cl})^{\mathrm{b}}\end{array}$ \\
\hline \multicolumn{5}{|c|}{$\begin{array}{l}\text { Age group-specific } \\
\text { analysis }\end{array}$} \\
\hline \multirow[t]{5}{*}{ All vaccines $^{c}$} & Ages 30 years and older & $2725 ; 1606 / 11557 ; 9706$ & $74(72-77)$ & $74(70-77)$ \\
\hline & 30-44 years & $1066 ; 291 / 3268 ; 1938$ & 75 (71-79) & $74(69-79)$ \\
\hline & $45-59$ years & $992 ; 722 / 4229 ; 3875$ & $76(71-80)$ & $76(71-80)$ \\
\hline & $60-74$ years & $410 ; 346 / 2412 ; 2277$ & $64(48-75)$ & $63(48-75)$ \\
\hline & 75 years and older & $255 ; 245 / 1639 ; 1613$ & $62(16-83)$ & $63(16-83)$ \\
\hline \multirow[t]{2}{*}{ Comirnaty $^{d}$} & $30-59$ years & $1640 ; 595 / 5546 ; 3856$ & $78(75-80)$ & $78(75-81)$ \\
\hline & 60 years and older & $449 ; 375 / 2735 ; 2574$ & $67(54-76)$ & $67(52-77)$ \\
\hline
\end{tabular}




\begin{tabular}{|c|c|c|c|c|}
\hline Vaxzevria $^{d}$ & $30-59$ years & $1141 ; 151 / 2294 ; 639$ & $66(58-73)$ & $66(58-73)$ \\
\hline & 60 years and older & 220;150/1073;915 & $62(45-74)$ & $65(48-76)$ \\
\hline \multirow[t]{2}{*}{ Spikevax $^{d}$} & $30-59$ years & $1071 ; 38 / 2268 ; 596$ & $91(87-93)$ & $91(87-94)$ \\
\hline & 60 years and older & $86 ; 20 / 387 ; 243$ & $81(66-90)$ & $83(64-92)$ \\
\hline \multirow[t]{2}{*}{$\begin{array}{l}\text { COVID-19 Vaccine } \\
\text { Janssen }^{\mathrm{e}}\end{array}$} & $30-59$ years & $1136 ; 217 / 2199 ; 621$ & $46(35-56)$ & $52(40-61)$ \\
\hline & 60 years and oldere & $99 ; 42 / 235 ; 101$ & & \\
\hline \multicolumn{5}{|l|}{$\begin{array}{l}\text { Chronic condition- } \\
\text { specific analysis }\end{array}$} \\
\hline \multirow[t]{4}{*}{ All vaccines $^{f}$} & $\begin{array}{l}\text { Presence of chronic } \\
\text { condition, } 30-59 \text { years }\end{array}$ & $331 ; 199 / 1604 ; 1305$ & $68(58-76)$ & $63(50-73)$ \\
\hline & $\begin{array}{l}\text { Absence of chronic } \\
\text { condition, 30-59 years }\end{array}$ & $1709 ; 807 / 5778 ; 4424$ & $75(72-78)$ & $77(73-80)$ \\
\hline & $\begin{array}{l}\text { Presence of chronic } \\
\text { condition, } 60 \text { years and } \\
\text { older }\end{array}$ & 289;266/1976;1908 & $55(24-74)$ & $58(27-76)$ \\
\hline & $\begin{array}{l}\text { Absence of chronic } \\
\text { condition, } 60 \text { years and } \\
\text { older }\end{array}$ & $367 ; 320 / 2049 ; 1960$ & $67(51-78)$ & $66(49-78)$ \\
\hline \multirow[t]{2}{*}{ Comirnaty } & $\begin{array}{l}\text { Presence of chronic } \\
\text { condition, } 30-59 \text { years }\end{array}$ & $257 ; 125 / 1190 ; 891$ & $72(62-80)$ & $69(56-78)$ \\
\hline & $\begin{array}{l}\text { Absence of chronic } \\
\text { condition, } 30-59 \text { years }\end{array}$ & $1383 ; 470 / 4329 ; 2946$ & $79(75-81)$ & $80(77-83)$ \\
\hline \multirow[t]{2}{*}{ Vaxzevria } & $\begin{array}{l}\text { Presence of chronic } \\
\text { condition, } 30-59 \text { years }\end{array}$ & $143 ; 20 / 381 ; 89$ & $54(20-74)$ & $50(11-72)$ \\
\hline & $\begin{array}{l}\text { Absence of chronic } \\
\text { condition, } 30-59 \text { years }\end{array}$ & $997 ; 131 / 1904 ; 543$ & $67(59-74)$ & $68(60-75)$ \\
\hline \multirow[t]{2}{*}{ Spikevax } & $\begin{array}{l}\text { Presence of chronic } \\
\text { condition, 30-59 years }\end{array}$ & $136 ; 5 / 464 ; 167$ & $94(85-98)$ & $94(83-98)$ \\
\hline & $\begin{array}{l}\text { Absence of chronic } \\
\text { condition, } 30-59 \text { years }\end{array}$ & $935 ; 33 / 1802 ; 429$ & $89(85-93)$ & $90(86-94)$ \\
\hline
\end{tabular}




\begin{tabular}{|l|l|r|r|r|}
\hline $\begin{array}{l}\text { COVID-19 Vaccine } \\
\text { Janssen }\end{array}$ & $\begin{array}{l}\text { Presence of chronic } \\
\text { condition, 30-59 years }\end{array}$ & $168 ; 46 / 423 ; 132$ & $25(-15-50)$ & $24(-25-53)$ \\
\hline & $\begin{array}{l}\text { Absence of chronic } \\
\text { condition, 30-59 years }\end{array}$ & $968 ; 171 / 1774 ; 489$ & $50(39-60)$ & $57(45-66)$ \\
\hline
\end{tabular}

${ }^{a}$ Adjusted by study site, swab date.

${ }^{b}$ Adjusted by study site, swab date, 10 -year age group, presence of chronic condition, sex.

c Six controls dropped from the 30-44 years (RO), four controls dropped from the 45-59 years (RO), and two cases dropped from the 75 years and older analysis (RO).

d Only countries included where at least one study participant in the age group for analysis had received the vaccine under study. Comirnaty: NL-CO, EN, ES, FR, HR, IE, NA, PT, RO, SC; Vaxzevria: NL-CO, EN, ES, FR, HR (30-59-year-olds only), IE, NA, PT, SC; Spikevax: NL-CO, ES, FR, HR (30-59-year-olds only), IE (30-59-year-olds only), NA, PT, RO (60 years and older only), SC (30-59-year-olds only); COVID-19 Vaccine Janssen: NL-CO, ES, FR, HR, IE, NA, PT

e VE was not computed for COVID-19 Vaccine Janssen among those aged 60 and older, as all countries but one dropped out of the analysis.

${ }^{f}$ Countries included in the 30-59 years old analysis: NL-CO, ES, FR, HR, NA, PT, SC; countries included in the 60 years and over analysis: NL-CO, EN, ES, FR, IE, NA, RO, SC

Comirnaty VE among those aged 30-59 by days between vaccination and onset of symptoms was 87\% (95\% Cl: 83-89) at 14-29 days and 65\% (95\% Cl: 56-71\%) at $\geq 90$ days ( $\mathrm{p}$-value test for trend $<0.001$ ) (Table 3). Using our modelling approach, the VE declined from 90\% (95\% Cl: 84-94) at 14 days to $61 \%(95 \% \mathrm{Cl}: 43-73)$ at 213 days (Figure 4$)$.

Comirnaty VE among those aged 60 years and older by days between vaccination and onset of symptoms was $65 \%(95 \% \mathrm{Cl}: 37-80)$ at $30-59$ days and $64 \%(95 \% \mathrm{Cl}: 44-77 \%)$ at $\geq 90$ days (Table 3). Sample size was too small to measure VE at 14-29 days (2 vaccinated cases and 30 controls, respectively). Using the modelling approach the VE declined from $89 \%$ (95\% $\mathrm{Cl}$ : -22-99) at 16 days to $50 \%$ (95\% Cl: 2-75) at 203 days (Figure 4).

Vaxzevria VE among those aged 30-59 years by days between vaccination and onset of symptoms was $72 \%(95 \% \mathrm{Cl} 52-83)$ at $14-29$ days and $65 \%$ (95\% Cl: 48-76) at 60-89 days (Table 3). Spikevax VE among those aged 30-59 years by days between vaccination and onset of symptoms was $98 \%$ (95\% Cl 93-100) at 14-29 days and 90\% (95\% Cl: 76-96) at 60-89 days (Table 3). COVID-19 Vaccine Janssen VE among those aged 30-59 years by days between vaccination and onset of symptoms was $50 \%(95 \% \mathrm{Cl}: 36-62)$ at 30-59 days and 52\% (95\% Cl: 33-66) at 60-89 days. Sample size was too small to measure COVID-19 Vaccine Janssen VE for 14-29 days and to measure VE among those vaccinated $\geq 90$ days for all vaccines except Comirnaty, and modelling was not 
attempted. Sample size was also too small to measure VE by time since vaccination among those aged 60 years and older for these vaccine brands.

Table 3: Effectiveness of complete COVID-19 vaccination among participants in the primary care and community I-MOVE-COVID-19 and ECDC VE study, by time since vaccination and vaccine product, Europe, July-August 2021

\begin{tabular}{|c|c|c|c|}
\hline \multicolumn{4}{|c|}{ Analysis by time since vaccination } \\
\hline $\begin{array}{l}\text { Brand, age group and time } \\
\text { since vaccination }\end{array}$ & Cases / controls & Crude VE $(95 \% \mathrm{Cl})^{\mathrm{a}}$ & Adjusted VE $(95 \% \mathrm{Cl})^{b}$ \\
\hline \multicolumn{4}{|l|}{ Comirnaty, age 30-59 years ${ }^{c}$} \\
\hline Unvaccinated & $1045 / 1684$ & & \\
\hline Vaccinated $14-29$ days & $123 / 1287$ & $87(84-89)$ & $87(83-89)$ \\
\hline Vaccinated 30-59 days & $261 / 1584$ & 75 (71-79) & $76(72-81)$ \\
\hline Vaccinated $60-89$ days & $60 / 335$ & $70(59-78)$ & $72(61-80)$ \\
\hline Vaccinated $\geq 90$ days & $151 / 647$ & $66(58-72)$ & $65(56-71)$ \\
\hline \multicolumn{4}{|l|}{ Comirnaty, age $60+$ years $^{c}$} \\
\hline Unvaccinated & $74 / 161$ & & \\
\hline Vaccinated $14-29$ days & $2 / 30$ & - & - \\
\hline Vaccinated $30-59$ days & $32 / 425$ & $67(42-81)$ & $65(37-80)$ \\
\hline Vaccinated $60-89$ days & $146 / 951$ & $65(49-76)$ & $66(48-78)$ \\
\hline Vaccinated $\geq 90$ days & 192/1159 & $66(51-76)$ & $64(44-77)$ \\
\hline \multicolumn{4}{|l|}{$\begin{array}{l}\text { Vaxzevria, age 30-59 } \\
\text { years }^{d}\end{array}$} \\
\hline Unvaccinated & $990 / 1655$ & & \\
\hline Vaccinated $14-29$ days & $21 / 107$ & $71(52-83)$ & $72(52-83)$ \\
\hline Vaccinated $30-59$ days & $79 / 320$ & $67(56-75)$ & $67(57-75)$ \\
\hline Vaccinated $60-89$ days & $42 / 162$ & $64(47-76)$ & $65(48-76)$ \\
\hline Vaccinated $\geq 90$ days & $9 / 50$ & - & - \\
\hline \multicolumn{4}{|l|}{ Spikevax, age 30-59 years ${ }^{\mathrm{e}}$} \\
\hline Unvaccinated & $1033 / 1672$ & & \\
\hline Vaccinated 14-29 days & $2 / 180$ & $98(92-100)$ & $98(93-100)$ \\
\hline Vaccinated 30-59 days & $19 / 285$ & $91(85-94)$ & $91(85-95)$ \\
\hline
\end{tabular}




\begin{tabular}{|l|c|c|c|}
\hline Vaccinated 60-89 days & $6 / 98$ & $89(75-96)$ & $90(76-96)$ \\
\hline Vaccinated $\geq 90$ days & $11 / 33$ & - & \\
\hline Janssen, age 30-59 years & & & \\
\hline Unvaccinated & & & - \\
\hline Vaccinated $14-29$ days & $19 / 61$ & - & $50(36-62)$ \\
\hline Vaccinated 30-59 days & $123 / 338$ & $46(32-57)$ & $52(33-66)$ \\
\hline Vaccinated 60-89 days & $70 / 205$ & $45(26-60)$ & - \\
\hline Vaccinated $\geq 90$ days & $5 / 17$ & - & \\
\hline
\end{tabular}

${ }^{a}$ Adjusted by study site, swab date.

${ }^{b}$ Adjusted by study site, swab date, 10 -year age group, presence of chronic condition, sex.

${ }^{c}$ Comirnaty: NL-CO, EN (30-59-year-old analysis only), ES, FR, HR, IE, NA, PT, RO (60 years and older analysis only), SC

d Vaxzevria: NL-CO, EN, ES, FR, HR, IE, NA, PT, SC

e Spikevax: NL-CO, ES, FR, IE, NA, PT, SC

fJanssen: NL-CO, ES, FR, HR, IE, NA, PT 
Figure 4: Effectiveness of Comirnaty vaccination among participants in the primary care/community I-MOVE-COVID-19 and ECDC VE study by days between dose of complete vaccine and onset of symptoms, and by age group, Europe, July-August 2021

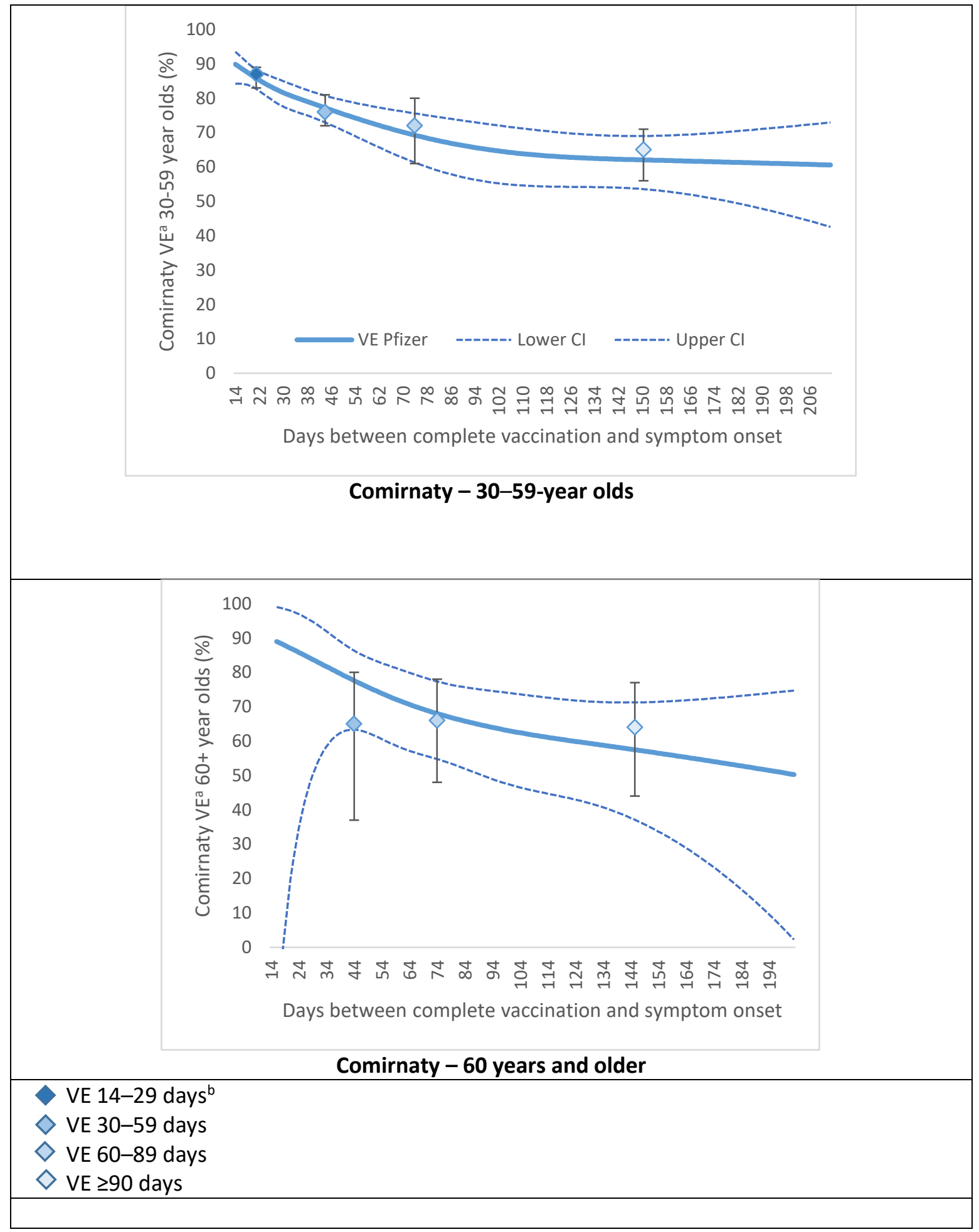

${ }^{a}$ Adjusted by study site, age group, sex, date of swab and presence of chronic condition.

b Sample size was too small to measure VE for 14-29 days for Comirnaty among those aged 60 years and older 
$\underline{\text { Sensitivity analysis }}$

Excluding the study site with self-selected participants (NL-CO), varying the imputed onset dates by 2 and 5 days, and restricting to those swabbed within 7 days of symptom onset, changed the VE point estimates by less than $3 \%$. Excluding the study site providing the most cases and controls (NA) resulted in lower sample size and not all stratified VE could be estimated, particularly for older age groups. VE point estimates among those aged $30-59$ years old differed by $\leq 8 \%$ for all estimates. For those aged 60 years and older, only VE for Comirnaty could be estimated, where the VE differed by $12 \%$, although confidence intervals overlapped (67\% (95\% Cl: $52-77)$ and $79 \%$ (95\% Cl: 54-91), including and excluding NA, respectively).

Varying the position of knots in time since vaccination analysis (equal intervals, different percentiles) changed the Akaike Information Criterion (AIC) by $<3$ points among those aged 30-59 years and $<4$ points among those aged 60 and older, and the functional forms remained the same.

\section{Discussion}

In this multicentre test-negative design study, including 14,282 patients, we measured VE for Comirnaty, Vaxzevria, Spikevax and Janssen vaccines against symptomatic SARS-CoV-2 infection in July and August 2021, thus restricting to a period where Delta variant circulation dominated.

VE was greater for mRNA vaccines than for the other vaccines among those aged 30-59 years, with Spikevax at 91\%, Comirnaty at 78\%, and Vaxzevria and COVID-19 Vaccine Janssen VE at 66\% and $52 \%$, respectively.

Among those aged 60 years and older, VE was 67\% (Comirnaty), 83\% (Spikevax) and 65\% (Vaxzevria). Sample size was too small to measure VE in this age group for COVID-19 Vaccine Janssen. Confidence intervals overlapped due to small sample size in this age group, and no comparison by vaccine brand was possible.

For the two mRNA vaccines, the point estimates were higher for Spikevax compared to Comirnaty in for the 30-59 and 60 years and older age groups, confidence intervals overlapped in the older age group.

Due to the observational nature of this study, comparison between vaccine brands should be made with caution, as different population groups (with varying levels of risk and exposure) may 
have been offered different vaccines at different times. Nevertheless, our results suggest a reduction greater than $50 \%$ in symptomatic SARS-CoV-2 infection by all vaccine brands across all adults aged 30 years upwards in July and August 2021 during Delta circulation dominance.

The Comirnaty VE among those aged 60 years and older was lower than in our previous publication among those aged 65 and older for the study period December 2020 to May 2021 (67\% vs. $87 \%$, respectively)(17). The differences in VE may be explained by the difference in predominant circulating variants between these periods (alpha variant vs. delta variant) and potential waning of the effect of the vaccine over time.

Our estimates are also similar to the VE against symptomatic infection in other observational studies during Delta circulation. In the UK, while the VE point estimate of Comirnaty was higher (88\% compared to our VE of $67-78 \%$ ), the VE for Vaxzevria was similar (67\% to $65-66 \%)(23)$. In Qatar, the VE of Comirnaty was lower (54\% compared to our $67-78 \%$ ) and the VE for Spikevax was similar (85\% compared to our 83-91\%), although the study design differed to ours (24).

We observed a lower VE point estimate among those presenting at least one chronic condition, compared to those without both for 30-59-year-olds and those aged 60 years and older. Precision was low in the older age group and the difference (14\%) was only statistically significant among those aged 30-59 years. Brand-specific VE by chronic conditions among those aged 30-69 suggest that for most brands, VE is higher among those without chronic conditions as observed in the overall study population. Brand-specific VE by presence of chronic condition was not available for other age groups due to limited sample size. Small differences between VE against infection among those with and without underlying chronic conditions has been suggested in a Danish study(25), and specifically for those immunocompromised, but not for any chronic condition in a UK study(26). Other studies did not observe differences in VE against infection among those with and without presence of chronic conditions $(27,28)$. Further studies presenting brand-specific VE by age group, including also estimates among those without chronic conditions as a comparator group are needed to add to the evidence of how the vaccines perform in different risk groups.

There was some evidence of decline in VE by time since vaccination among those aged $30-59$ for Comirnaty (ranging from $87 \%$ at $14-29$ days to $65 \%$ at $90+$ days). Modelling suggested the VE declined to $61 \%$ after 200 days. Among those aged 60 years and older, VE point estimates differed minimally between 30-59 days and 90+ days (63-66\%), using an approach of measuring VE by time since vaccination by cutoffs, but precision was low. Modelling VE by time since vaccination as 
a continuous variable indicated some potential decline over time, but confidence intervals are also compatible with no decline. Sample size in this age group was too low to measure VE by time since vaccination for Spikevax and Vaxzevria. It is worth noting that this study does not include the frail elderly, e.g., those who present directly to hospital or are care facility residents.

Using a modelling approach to measuring VE by days since vaccination provides added value compared to the stratified approach, as we do not lose information by transforming a continuous variable into a categorical one. However, the modelling approach may not be adequate in the tails of the model, due to sparse data, as can be seen by the very wide confidence intervals around the model among those aged 60 years and older. This may explain why the stratified estimates do not match the ones in the model well up to 60 days between vaccination and symptom onset.

In the UK, a smaller decline of Comirnaty VE against infection for younger adults and of both mRNA vaccines for adults in Canada was observed (both $12 \%$ compared to our $22 \%$ over a similar time since vaccination period)(14,16). A larger decline in Comirnaty VE against Delta infection was observed among healthcare workers in the US (93\% to 53\% over 5 months) (29). Similar to our study, no decline was observed for Vaxzevria among this age group in the UK and at most a $12 \%$ decline among adults in Canada. No decline was observed in the UK for Spikevax, however a 14\% decline was observed in the US (30).

To disentangle the decline in VE by time since vaccination from variant-related reduction in VE over time, effects of vaccine brands given at different times and differences in age-specific VE, it is important to examine the data and measure VE by time since vaccination by age group, and vaccine brand within a period of stable variant circulation. While this is possible to a certain extent in our study, sample size becomes limited and some results may be spurious. We will endeavour to repeat these analyses going forward with larger sample size.

Study design-specific issues and mechanism of protection of the vaccine can hide or exacerbate changes in VE by time since vaccination (31). A spurious waning in the vaccine effect can be observed if the vaccine provides only partial protection, due to differential depletion of susceptibles in unvaccinated and vaccinated groups (32). In the context of a high baseline VE, which is what we observe here, the bias may be minimal (33).

Those vaccinated earlier may belong to specific groups with different risks of exposure and susceptibility to the virus than those vaccinated late. Adjusting for presence of chronic condition, time and age in our analyses may not be sufficient. Measuring age group and brand-specific VE by 
time since vaccination by vaccine cohort (vaccinated early, middle or late) may provide additional information.

Our study is subject to several limitations. While many study sites sequence the whole or partial genome of viruses, the proportion sequenced overall did not allow a variant-specific VE analysis. To overcome this limitation, we restricted the study period to July-August 2021, a period in which we know the Delta variant dominated in the European sites participating in our study. Among the 130 viruses sequenced, $91 \%$ belonged to the SARS-CoV-2 Delta variant, indicating that the vast majority of viruses in the study were likely to belong to the Delta variant. The proportion of viruses sequenced is low in our study, the main reason for this is the difficulty of being able to match the clinical-epidemiological data with the virological data.

The vaccine coverage was high in July and August in many European countries, particularly among older age groups. Apart from affecting our results in terms of precision, patients who are unvaccinated may be different from vaccinated patients in terms of exposure to the virus, thus violating a key precondition of VE studies (34). Inclusion of confounders in the VE model can help adjust for different exposures. Including measures of clinical extremely vulnerable people may help overcome confounding (35). Confounding factors relating to behaviour are difficult to collect, although ongoing prospective cohort studies in special populations (e.g., healthcare workers) may help us understand what confounding to expect.

We use a multicentre study design, and aim to reduce heterogeneity with study sites adapting the same generic protocol. The study site in Navarra, Spain, dominates in terms of sample size, as it provides data from all symptomatic patients tested using their comprehensive surveillance using electronic medical records/registries. Excluding Navarra from the analysis reduces precision, however the point estimates for all analyses remain $<10 \%$ different in terms of absolute VE, with the exception of Comirnaty VE among those aged $\geq 60$ years, where excluding Navarra resulted in a point estimate greater by $12 \%$, but due to sample size confidence intervals overlapped.

Our study has several strengths. Selection bias can be a problem with observational studies, particularly in analyses involving electronic medical records. In our study, in all study sites, but one, all or a systematic sample of patients are selected for inclusion. The swabbing procedure is known, reducing the risk of selection bias. Additionally, the test-negative design aims to minimise selection bias by adjusting for healthcare-seeking behaviour. Omitting the one study with selfselected patients changes the VE by $<2 \%$ overall, by age group and vaccine brand. 


\section{Conclusions}

Our study provides evidence of 52-91\% protection of complete dose vaccination against the Delta variant among those aged 30 years and older for four vaccine brands. The results also indicate a lower VE among those with presence of chronic condition among those aged 30-59 for some vaccine brands. While our study suggests some waning of the vaccine effect with increased time since vaccination, we observed sustained protection above $60 \%$ up to 200 days post vaccination for Comirnaty. Further studies are needed to better analyse this observed decline in VE. We will continue the analysis of VE by days since vaccination and onset within the I-MOVE-COVID-19 and ECDC network study as more data come in.

High complete vaccine coverage in the primary series of vaccination is important as it provides good direct protection of individuals against symptomatic SARS-CoV-2 infection as illustrated by this and other studies, as well as providing protection against severe disease $(16,36)$. 


\section{References}

1. European Centre for Disease Prevention and Control. COVID-19 situation update worldwide, as of week 44, updated 11 November 2021 [Internet]. 2021 [cited 2021 Nov 15]. Available from: https://www.ecdc.europa.eu/en/geographical-distribution-2019-ncov-cases

2. European Centre for Disease Prevention and Control (ECDC), World Health Organization Regional Office for Europe (WHO/Europe). Assessing SARS-CoV-2 circulation, variants of concern, non-pharmaceutical interventions and vaccine rollout in the EU/EEA, 16th update [Internet]. European Centre for Disease Prevention and Control; 2021 Sep [cited 2021 Oct 28]. Available from: https://www.ecdc.europa.eu/sites/default/files/documents/covid-19rapid-risk-assessment-16th-update-september-2021.pdf

3. Nextstrain. Genomic epidemiology of novel coronavirus - Europe-focused subsampling [Internet]. [cited 2021 Oct 30]. Available from: https://nextstrain.org/ncov/gisaid/europe

4. Shu Y, McCauley J. GISAID: Global initiative on sharing all influenza data - from vision to reality. Euro Surveill. 2017 Mar 30;22(13):30494.

5. European Medicines Agency. COVID-19 vaccines [Internet]. Available from: https://www.ema.europa.eu/en/human-regulatory/overview/public-healththreats/coronavirus-disease-covid-19/treatments-vaccines/covid-19-vaccines

6. Baden LR, El Sahly HM, Essink B, Kotloff K, Frey S, Novak R, et al. Efficacy and Safety of the mRNA-1273 SARS-CoV-2 Vaccine. N Engl J Med. 2021 Feb 4;384(5):403-16.

7. Polack FP, Thomas SJ, Kitchin N, Absalon J, Gurtman A, Lockhart S, et al. Safety and Efficacy of the BNT162b2 mRNA Covid-19 Vaccine. N Engl J Med. 2020 Dec 31;383(27):2603-15.

8. Sadoff J, Gray G, Vandebosch A, Cárdenas V, Shukarev G, Grinsztejn B, et al. Safety and Efficacy of Single-Dose Ad26.COV2.S Vaccine against Covid-19. N Engl J Med. 2021 Jun $10 ; 384(23): 2187-201$.

9. Voysey M, Clemens SAC, Madhi SA, Weckx LY, Folegatti PM, Aley PK, et al. Safety and efficacy of the ChAdOx1 nCoV-19 vaccine (AZD1222) against SARS-CoV-2: an interim analysis of four randomised controlled trials in Brazil, South Africa, and the UK. The Lancet. 2021 Jan;397(10269):99-111.

10. Rosenberg ES, Dorabawila V, Easton D, Bauer UE, Kumar J, Hoen R, et al. COVID-19 Vaccine Effectiveness by Product and Timing in New York State [Internet]. Infectious Diseases (except HIV/AIDS); 2021 Oct [cited 2021 Oct 28]. Available from:

http://medrxiv.org/lookup/doi/10.1101/2021.10.08.21264595

11. Istituto Superiore di Sanita. Impact of COVID-19 vaccination on the risk of SARS-CoV-2 infection and hospitalization and death in Italy [Internet]. Rome, Italy: Istituto Superiore di Sanita; [cited 2021 Oct 28]. Available from: https://www.iss.it/documents/20126/0/report_on_vaccine_effectiveness_Italy+\%281\%29.p $\mathrm{df} / 53 \mathrm{~d} 71 \mathrm{dc2}-\mathrm{c8c5}-24 \mathrm{c1}-3467-705 \mathrm{a} 8587 \mathrm{a339} \mathrm{t}=1633529045681$ 
12. de Gier B, Andeweg S, Backer JA, RIVM COVID-19 surveillance and epidemiology team, Hahné SJM, van den Hof S, et al. Vaccine effectiveness against SARS-CoV-2 transmission to household contacts during dominance of Delta variant (B.1.617.2), August-September 2021, the Netherlands [Internet]. Epidemiology; 2021 Oct [cited 2021 Oct 28]. Available from: http://medrxiv.org/lookup/doi/10.1101/2021.10.14.21264959

13. Martínez-Baz I, Miqueleiz A, Casado I, Navascués A, Trobajo-Sanmartín C, Burgui C, et al. Effectiveness of COVID-19 vaccines in preventing SARS-CoV-2 infection and hospitalisation, Navarre, Spain, January to April 2021. Eurosurveillance [Internet]. 2021 May 27 [cited 2021 Jun 21];26(21). Available from: https://www.eurosurveillance.org/content/10.2807/15607917.ES.2021.26.21.2100438

14. N Andrews, E Tessier, J Stowe, C Gower, F Kirsebom, R Simmons, et al. Preprint: Vaccine effectiveness and duration of protection of Comirnaty, Vaxzevria and Spikevax against mild and severe COVID-19 in the UK. [cited 2021 Oct 30]; Available from:

https://khub.net/documents/135939561/338928724/Vaccine+effectiveness+and+duration+ of+protection+of+covid+vaccines+against+mild+and+severe+COVID19+in+the+UK.pdf/10dcd99c-0441-0403-dfd8-11ba2c6f5801

15. Poukka E, Baum U, Palmu AA, Lehtonen TO, Salo H, Nohynek H, et al. Cohort study of Covid19 vaccine effectiveness among healthcare workers in Finland, December 2020 - October 2021 [Internet]. Infectious Diseases (except HIV/AIDS); 2021 Nov [cited 2021 Nov 24]. Available from: http://medrxiv.org/lookup/doi/10.1101/2021.11.03.21265791

16. Skowronski DM, Setayeshgar S, Febriani Y, Ouakki M, Zou M, Talbot D, et al. Two-dose SARSCoV-2 vaccine effectiveness with mixed schedules and extended dosing intervals: testnegative design studies from British Columbia and Quebec, Canada [Internet]. Infectious Diseases (except HIV/AIDS); 2021 Oct [cited 2021 Oct 30]. Available from: http://medrxiv.org/lookup/doi/10.1101/2021.10.26.21265397

17. Kissling E, Hooiveld M, Sandonis Martín V, Martínez-Baz I, William N, Vilcu A-M, et al. Vaccine effectiveness against symptomatic SARS-CoV-2 infection in adults aged 65 years and older in primary care: I-MOVE-COVID-19 project, Europe, December 2020 to May 2021.

Eurosurveillance [Internet]. $2021 \mathrm{Jul} 22$ [cited 2021 Oct 28];26(29). Available from: https://www.eurosurveillance.org/content/10.2807/1560-7917.ES.2021.26.29.2100670

18. Epiconcept. COVID-19 vaccine effectiveness at primary care level in Europe: I-MOVE-COVID19 generic protocol [Internet]. [cited 2021 Jun 22]. Available from:

https://www.imoveflu.org/wp-content/uploads/2021/05/I-MOVE-COVID-19-primary-careCOVID-19-vaccine-effectiveness-protocol-v2.3.pdf

19. Jackson ML, Nelson JC. The test-negative design for estimating influenza vaccine effectiveness. Vaccine. 2013 Apr 19;31(17):2165-8.

20. Wellcome Sanger Institute. COVID-19 Genomic Surveillance [Internet]. COVID-19 Genomic Surveillance. [cited 2021 Oct 28]. Available from: https://covid19.sanger.ac.uk

21. European Centre for Disease Prevention and Control. Data on SARS-CoV-2 variants in the EU/EEA [Internet]. [cited 2021 Oct 28]. Available from: https://www.ecdc.europa.eu/en/publications-data/data-virus-variants-covid-19-eueea 
22. Harrell FE. Regression modeling strategies: with applications to linear models, logistic regression, and survival analysis. New York: Springer; 2001. 568 p. (Springer series in statistics).

23. Lopez Bernal J, Andrews N, Gower C, Gallagher E, Simmons R, Thelwall S, et al. Effectiveness of Covid-19 Vaccines against the B.1.617.2 (Delta) Variant. N Engl J Med. 2021 Aug 12;385(7):585-94.

24. Tang $P$, Hasan MR, Chemaitelly H, Yassine HM, Benslimane FM, Khatib HAA, et al. BNT162b2 and mRNA-1273 COVID-19 vaccine effectiveness against the Delta (B.1.617.2) variant in Qatar [Internet]. Epidemiology; 2021 Aug [cited 2021 Oct 30]. Available from: http://medrxiv.org/lookup/doi/10.1101/2021.08.11.21261885

25. Emborg H-D, Valentiner-Branth P, Schelde AB, Nielsen KF, Gram MA, Moustsen-Helms IR, et al. Vaccine effectiveness of the BNT162b2 mRNA COVID-19 vaccine against RT-PCR confirmed SARS-CoV-2 infections, hospitalisations and mortality in prioritised risk groups [Internet]. Public and Global Health; 2021 Jun [cited 2021 Nov 25]. Available from: http://medrxiv.org/lookup/doi/10.1101/2021.05.27.21257583

26. Whitaker HJ, Tsang RS, Byford R, Andrews NJ, Sherlock J, Pillai PS, et al. Pfizer-BioNTech and Oxford AstraZeneca COVID-19 vaccine effectiveness and immune response among individuals in clinical risk groups. [cited 2021 Nov 25]; Available from: https://khub.net/documents/135939561/430986542/RCGP+VE+riskgroups+paper.pdf/a6b5 4cd9-419d-9b63-e2bf-5dc796f5a91f

27. Thompson MG, Stenehjem E, Grannis S, Ball SW, Naleway AL, Ong TC, et al. Effectiveness of Covid-19 Vaccines in Ambulatory and Inpatient Care Settings. N Engl J Med. 2021 Oct 7;385(15):1355-71.

28. Dagan N, Barda N, Kepten E, Miron O, Perchik S, Katz MA, et al. BNT162b2 mRNA Covid-19 Vaccine in a Nationwide Mass Vaccination Setting. N Engl J Med. 2021 Apr 15;384(15):141223.

29. Tartof SY, Slezak JM, Fischer H, Hong V, Ackerson BK, Ranasinghe ON, et al. Effectiveness of mRNA BNT162b2 COVID-19 vaccine up to 6 months in a large integrated health system in the USA: a retrospective cohort study. The Lancet. 2021 Oct;398(10309):1407-16.

30. Bruxvoort KJ, Sy LS, Qian L, Ackerson BK, Luo Y, Lee GS, et al. Effectiveness of mRNA-1273 against Delta, Mu, and other emerging variants [Internet]. Infectious Diseases (except HIV/AIDS); 2021 Oct [cited 2021 Oct 30]. Available from:

http://medrxiv.org/lookup/doi/10.1101/2021.09.29.21264199

31. Halloran ME, Longini IM, Struchiner CJ. Design and analysis of vaccine studies. New York: Springer; 2010. 387 p. (Statistics for biology and health).

32. World Health Organisation. Evaluation of COVID-19 vaccine effectiveness - Interim guidance [Internet]. World Health Organization; 2021 [cited 2021 Oct 31]. Available from: https://www.who.int/publications/i/item/WHO-2019-nCoV-vaccine_effectivenessmeasurement-2021.1 
33. Kahn R, Schrag SJ, Verani JR, Lipsitch M. Identifying and alleviating bias due to differential depletion of susceptible people in post-marketing evaluations of COVID-19 vaccines [Internet]. Epidemiology; 2021 Jul [cited 2021 Oct 31]. Available from:

http://medrxiv.org/lookup/doi/10.1101/2021.07.15.21260595

34. Greenwood M, Yule GU. The Statistics of Anti-typhoid and Anti-cholera Inoculations, and the Interpretation of such Statistics in general. Proc R Soc Med. 1915;8(Sect Epidemiol State Med):113-94.

35. Andrews N. High vaccine coverage: Are unvaccinated at the same risk of exposure to SARSCoV-2 as the vaccinated? How can we measure this and implications for studies? ECDC VE study workshop; 2021 Oct 15.

36. Martínez-Baz I, Trobajo-Sanmartín C, Miqueleiz A, Guevara M, Fernández-Huerta M, Burgui C, et al. Product-specific COVID-19 vaccine effectiveness against secondary infection in close contacts, Navarre, Spain, April to August 2021. Eurosurveillance [Internet]. 2021 Sep 30 [cited 2021 Oct 28];26(39). Available from:

https://www.eurosurveillance.org/content/10.2807/1560-7917.ES.2021.26.39.2100894 
Conflict of interest: Professor de Lusignan has received grants not directly relating to this work, from AstraZeneca, GSK, Sanofi, Seqirus and Takeda for vaccine-related research and has been a member of advisory boards for AstraZeneca, Sanofi and Seqirus.

Funding statement: This project has received funding from the European Union's Horizon 2020 research and innovation programme under grant agreement No 101003673.

This project received funding from the European Centre for Disease Prevention and Control (ECDC) under the contract 11486 .

\section{Acknowledgements}

Thank you to colleagues that are part of the I-MOVE-COVID-19 and ECDC VE networks, with study starts after August 2021 and not included in this article: Krisztina Horvath (Semmelweis University, Hungary), Beatrix Oroszi (Semmelweis University, Hungary), and Nathalie Bossuyt (Sciensano, Belgium).

Special thanks to Alain Moren, for critical review of the manuscript and fruitful discussions.

All study teams are very grateful to all patients, general practitioners, paediatricians, laboratory teams, and regional epidemiologists who have contributed to the studies.

Participating laboratories submitted their sequences to GISAID (www.gisaid.org) for easy sharing with the central laboratory in Madrid.

\section{I-MOVE-COVID-19 and ECDC primary care study team (in addition to authors above)}

\section{Croatia}

Katica Čusek Adamić, Institute of Public Health, Varaždin County, Varaždin, Croatia Ivana Ferenčak, Croatian Institute of Public Health, Zagreb, Croatia Bernard Kaić, Croatian Institute of Public Health, Zagreb, Croatia Mirjana Lana Kosanović Ličina, "Andrija Stampar" Teaching Institute of Public Health, Zagreb, Croatia

Danijela Lakošeljac, Teaching Institute of Public Health, Primorje-Gorski kotar County, Rijeka, Croatia

Ivana Mihin Huskić, Teaching Institute of Public Health, Osijek-Baranja County, Osijek, Croatia Diana Nonković, Teaching Institute for Public Health, Split-Dalmatia County, Split, Croatia

\section{England}

Nick Andrews, Public Health England, London, England 
Jamie Lopez Bernal, Public Health England, London, England

Joanna Ellis, Public Health England, London, England

Heather Whitaker, Public Health England, London, England

\section{France}

Thierry Blanchon - Sorbonne Université, INSERM, Institut Pierre Louis d'épidémiologie et de Santé Publique (IPLESP UMRS 1136), Paris, France

Caroline Guerrisi - Sorbonne Université, INSERM, Institut Pierre Louis d'épidémiologie et de Santé Publique (IPLESP UMRS 1136), Paris, France

Titouan Launay - Sorbonne Université, INSERM, Institut Pierre Louis d'épidémiologie et de Santé Publique (IPLESP UMRS 1136), Paris, France

Shirley Masse - Laboratoire de Virologie, Université de Corse-Inserm, Corte, France Sylvie van der Werf, Unité de Génétique Moléculaire des Virus à ARN, UMR 3569 CNRS, Université Paris Diderot SPC, Institut Pasteur, Paris; CNR des virus des infections respiratoires, Institut Pasteur, Paris, France

Vincent Enouf, Unité de Génétique Moléculaire des Virus à ARN, UMR 3569 CNRS, Université Paris Diderot SPC, Institut Pasteur, Paris; CNR des virus des infections respiratoires, Institut Pasteur, Paris, France

\section{Ireland}

John Cuddihy, Health Service Executive-Health Protection Surveillance Centre, Ireland Lois O'Connor, Health Service Executive-Health Protection Surveillance Centre, Ireland Adele McKenna, Health Service Executive-Health Protection Surveillance Centre, Ireland Michael Joyce, Irish College of General Practitioners, Ireland Cillian de Gascun, Virus Reference Laboratory, University College Dublin, Ireland Joanne Moran, National Virus Reference Laboratory, University College Dublin, Ireland

\section{The Netherlands}

Rianne van Gageldonk-Lafeber, National Institute for Public Health and the Environment (RIVM), Bilthoven, the Netherlands

Susan J. Hahné, National Institute for Public Health and the Environment (RIVM), Bilthoven, the Netherlands

Hester E. de Melker, National Institute for Public Health and the Environment (RIVM), Bilthoven, the Netherlands

Ewout B. Fanoy, Department of Infectious Disease Control, Public Health Service Rotterdam-

Rijnmond (GGD), Rotterdam, the Netherlands

Stijn Raven, Department of Infectious Disease Control, Public Health Service Region Utrecht (GGD), Utrecht, the Netherlands

Marit Middeldorp, National Institute for Public Health and the Environment (RIVM), Bilthoven, the Netherlands

\section{Portugal}

Irina Kislaya, Departamento de Epidemiologia, Instituto Nacional de Saúde Dr. Ricardo Jorge, Lisbon, Portugal

Baltazar Nunes, Departamento de Epidemiologia, Instituto Nacional de Saúde Dr. Ricardo Jorge, Lisbon, Portugal 
Rita Roquete, Departamento de Epidemiologia, Instituto Nacional de Saúde Dr. Ricardo Jorge, Lisbon, Portugal

Adriana Silva, Departamento de Epidemiologia, Instituto Nacional de Saúde Dr. Ricardo Jorge, Lisbon, Portugal

Aryse Melo, Departamento de Doenças Infeciosas, Instituto Nacional de Saúde Dr. Ricardo Jorge, Portugal

Inês Costa, Departamento de Doenças Infeciosas, Instituto Nacional de Saúde Dr. Ricardo Jorge, Portugal

Nuno Verdasca, Departamento de Doenças Infeciosas, Instituto Nacional de Saúde Dr. Ricardo Jorge, Portugal

Patrícia Conde, Departamento de Doenças Infeciosas, Instituto Nacional de Saúde Dr. Ricardo Jorge, Portugal

Amélia Soeiro, Departamento de Doenças Infeciosas, Instituto Nacional de Saúde Dr. Ricardo Jorge, Portugal

\section{Romania}

Mihai Maria Elena, Cantacuzino National Military Medical Institute for Research and Development, Romania

Bistriceanu Iulia, Cantacuzino National Military Medical Institute for Research and Development, Romania

Ivanciuc Alina, Cantacuzino National Military Medical Institute for Research and Development, Romania

Dintoi Diana, Cantacuzino National Military Medical Institute for Research and Development, Romania

Pascu Catalina, Cantacuzino National Military Medical Institute for Research and Development, Romania

Jidovu Adrian, Cantacuzino National Military Medical Institute for Research and Development, Romania

\section{Scotland}

Debbie Sigerson, Public health Scotland, Glasgow, Scotland

Diogo FP Marques, Public health Scotland, Glasgow, Scotland

Anna Molesworth, Public health Scotland, Glasgow, Scotland

Leanne Quinn, Public health Scotland, Glasgow, Scotland

Miranda Leyton, Public health Scotland, Glasgow, Scotland

Selin Campbell, Public health Scotland, Glasgow, Scotland

Janine Thoulass, Public health Scotland, Glasgow, Scotland

Jim McMenamin, Public health Scotland, Glasgow, Scotland

\section{Spain}

Inmaculada Casas Flecha, National Centre for Microbiology, Institute of Health Carlos III, Madrid, Spain

Ana Martínez Mateo, S General de Vigilancia y Respuesta a Emergencias de Salud Pública, Agencia de Salud Pública, Catalunya and CIBER Epidemiologia y Salud Pública (CIBERESP), Spain Daniel Castrillejo, Servicio de Epidemiología, Dirección General de Salud Pública y Consumo, Melilla, Spain 
Eva María Martínez Ochoa, Servicio de Epidemiología y Prevención Sanitaria, Dirección General de Salud Pública, Consumo y Cuidados, La Rioja, Spain

Carmen Quiñones Rubio, Servicio de Epidemiología y Prevención Sanitaria, Dirección General de Salud Pública, Consumo y Cuidados, La Rioja, Spain

Concepción Delgado-Sanz, National Centre for Epidemiology, Institute of Health Carlos III, Madrid, Spain, Consortium for Biomedical Research in Epidemiology and Public Health (CIBERESP), Institute of Health Carlos III, Madrid, Spain

Jesús Oliva. National Centre for Epidemiology, Institute of Health Carlos III, Madrid, Spain, Consortium for Biomedical Research in Epidemiology and Public Health (CIBERESP), Institute of Health Carlos III, Madrid, Spain

Ana Miqueleiz, Hospital Universitario de Navarra, Pamplona, Spain Ana Navascués, Hospital Universitario de Navarra, Pamplona, Spain Camino Trobajo-Sanmartín, Hospital Universitario de Navarra, Pamplona, Spain Carmen Ezpeleta, Hospital Universitario de Navarra, Pamplona, Spain Paula López Moreno, Servicio Navarro de Salud-Osasunbidea, Pamplona, Spain Javier Gorricho, Servicio Navarro de Salud-Osasunbidea, Pamplona, Spain Eva Ardanaz, Instituto de Salud Pública y Laboral de Navarra-IdiSNA-CIBERESP, Pamplona, Spain Fernando Baigorria, Instituto de Salud Pública y Laboral de Navarra-IdiSNA-CIBERESP, Pamplona, Spain

Aurelio Barricarte, Instituto de Salud Pública y Laboral de Navarra-IdiSNA-CIBERESP, Pamplona, Spain

Cristina Burgui, Instituto de Salud Pública y Laboral de Navarra-IdiSNA-CIBERESP, Pamplona, Spain

Enrique de la Cruz, Instituto de Salud Pública y Laboral de Navarra-IdiSNA-CIBERESP, Pamplona, Spain

Nerea Egüés, Instituto de Salud Pública y Laboral de Navarra-IdiSNA-CIBERESP, Pamplona, Spain Manuel García Cenoz, Instituto de Salud Pública y Laboral de Navarra-IdiSNA-CIBERESP, Pamplona, Spain

Marcela Guevara, Conchi Moreno-Iribas, Instituto de Salud Pública y Laboral de Navarra-IdiSNACIBERESP, Pamplona, Spain

Carmen Sayón, Instituto de Salud Pública y Laboral de Navarra-IdiSNA-CIBERESP, Pamplona, Spain

ECDC

Pasi Penttinen, European Centre for Disease Prevention and Control, Stockholm, Sweden Christiana Carstairs, European Centre for Disease Prevention and Control, Stockholm, Sweden 